\title{
Adaptive optics methods in gravitational wave interferometric detectors, a perspective
}

\author{
L. Aiello, ${ }^{a}$ E. Cesarini, ${ }^{b}$ V. Fafone,${ }^{c}$ M. Lorenzini ${ }^{*}{ }^{a}$ D. Lumaca, ${ }^{c}$ Y. Minenkov, ${ }^{d}$ I. \\ Nardecchia, ${ }^{c}$ A. Rocchi ${ }^{d}$ and V. Sequino ${ }^{c}$ \\ a Gran Sasso Science Institute, I-67100 L'Aquila, Italy \\ b Centro Studi e Ricerche Enrico Fermi, I-00184 Roma, Italy \\ c Università degli Studi di Roma Tor Vergata, I-00133 Roma, Italy \\ d INFN, Sezione di Roma Tor Vergata, I-00133 Roma, Italy \\ E-mail: matteo.lorenzinieroma2.infn.it
}

\begin{abstract}
The performance of present and future gravitational wave detectors is limited by fundamental factors, such as thermal noise, seismic or newtonian noise and quantum nature of light. Besides, technological factors impact the reach of advanced detectors in that upgrade strategies are limited by state-of-art performances. In the realm of optics, the quantum limit to sensitivity will be addressed by injecting higher laser power and by exploiting the capabilities of squeezed light. In turn, technological efforts in the preparation of suitable optics able to meet more and more demandig requirements are ongoing. Moreover, solutions to mitigate the effect of known showstoppers such as parametric instablities are being studied.

The present day strategy to correct for residual cold defects in the core optics and to counteract the thermal effects due to power absorption is embedded in a set of sensors and actuators integrated in the Advanced Virgo design, the so called Thermal Compensation System (TCS). This system is designed to be focused on the needs of high power operation of the detector, nonetheless it is highly versatile and can deal with foreseen and unexpected issues. We discuss the features of the TCS with emphasis on its versatility and portability to upgraded detectors; we also present the status of the R\&D activity in the Tor Vergata labs, highlighting new applications where the methods of TCS can have a relevant impact, such as adaptive mode matching for squeezing and damping of parametric instabilities.
\end{abstract}

GRAvitational-waves Science\& technology Symposium - GRASS2018

1-2 March 2018

Palazzo Moroni, Padova (Italy)

${ }^{*}$ Speaker. 


\section{Introduction}

In the years after the first epochal detection of gravitational waves (GW) emitted by a binary black hole merger (GW150914 [1]) the Advanced LIGO [2] and Advanced Virgo [3] interferometric antennae collected a number of observed events, mostly merging binary black holes but also a coalescence of binary neutron stars (GW170817). The network of such detectors allowed a precise reconstruction of the sky position of the source, and particularly in the case of GW170817 it has been possible to identify the optical counterpart, shredding light on the phenomenon of short $\gamma$ ray bursts [4]. After this successful phase, the advanced detectors are now undergoing a series of planned upgrades to further enhance the sensitivity and become full partners in the era of multimessenger astronomy. Among other upgrades, advanced detectors feature an increase of the laser source power to reduce the shot noise limit at medium and high frequencies. In the case of Advanced Virgo, a design source power of $125 \mathrm{~W}$ is foreseen. Due to the high finesse and to the additional gain in the recycled configuration, the power deposited on the mirrors will range up to $200 \mathrm{~mW}$ with coating absorption at $0.3 \mathrm{ppm}$.

Thermally driven dioptric power in the test masses will induce a mismatch of both carrier and sideband fields in the resonant cavities. Moreover, a radius of curvature (RoC) mismatch will appear in the cavity mirrors due to the thermoelastic deformation of the mass surface. Among several others, the prominent undesired effect of these aberrations is a drop of the recycling gain for the sideband fields, eventually leading to an uncontrollable detector. A way out of this problem consists in the development of an integrated adaptive optics system able to detect and counteract the deviations from the design layout of the interferometer [5].

The optical layout of the recycling cavity in Advanced Virgo is close to degeneracy [6], making the cold detector status very sensitive to imperfections in the optics. A versatile aberration compensation has the further advantage of permitting the recovery of distortions coming from cold defects (substrate inhomogeneities, RoC deviations, point absorbers and so on), resulting in an increased robustness of the overall behaviour of the interferometer.

\section{The Thermal Compensation System}

As part of the design of Advanced Virgo, the Thermal Compensation System [5, 6] is conceived to tackle the aberration budget coming both from cold defects and from thermally driven effects. TCS features both wavefront sensing and actuation, so that it can be operated as a loop keeping the detector at the optimal working point. However, the facilities of the TCS are designed in view of a wide class of possible operations during the instrument commissioning. Examples of possible applications of TCS methods are adaptive matching of injected squeezing for GW detectors and reduction of round trip losses in filter cavities. As a reference, a scheme of the TCS parts integrated in the Advanced Virgo layout is shown in figure 1.

\subsection{Wavefront sensing}

Since thermal effects will be mostly relevant for long-cavity mirrors, for which the power absorbed is maximum, wavefront sensing has been designed to operate in transmission through the input optics and in reflection onto all the high reflectivity surfaces of the mirrors. 


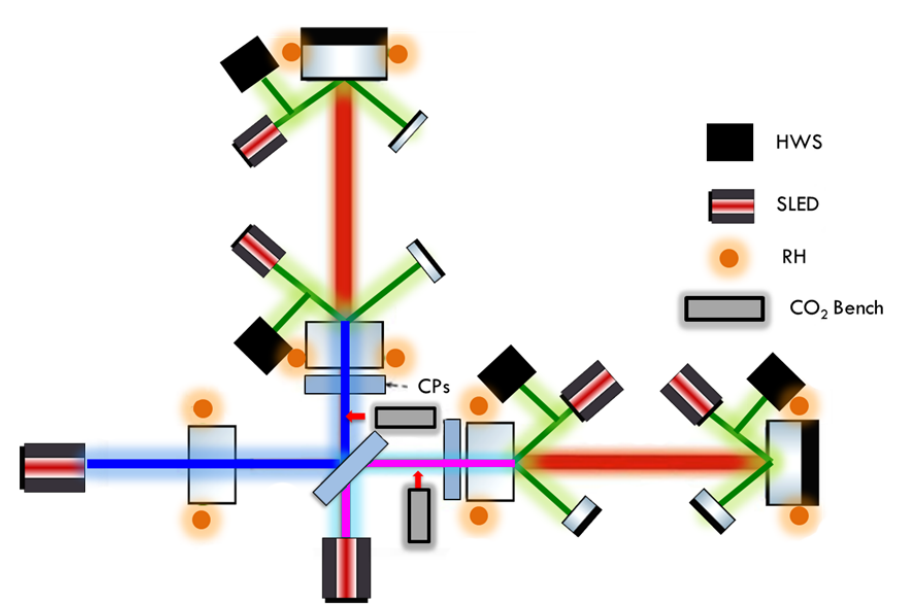

Figure 1: Scheme of the Thermal Compensation System of Advanced Virgo, superimposed on the optical layout of the detector. Blue and magenta beams represent the two HWS probe beams in transmission, while the actual HWS is omitted for the sake of clarity.

The phase distortion on the mirror is sampled by a probe beam and then reported to a conjugate plane by using an afocal optical system. The afocal system works as a beam shrinker, allowing the probe beam to match the size of sampled optics (tens of $\mathrm{cm}$ ) and then to be reduced to the optimal size for the wavefront sensing. The choice of a SLED (Superluminescent Light Emitting Diode) source as probe beam was driven by the need of avoiding diffraction effects.

Hartmann wavefront sensors (HWS, [7]) are employed to extract the phase information of the outcoming probe beam. In the HWS image plane, the beam is sampled by an array of many small holes (the Hartmann plate) and then projected on a CCD at a distance $L$. Each plate hole produces a spot on the CCD plane and an online software finds the positions $P_{i}$ of all the spot centroids. When the phase is changing, the displacement of $P_{i}$ tracks the change in wavefront slope on each hole. The wavefront is then obtained by integration of the gradient field output by the HWS. HWS is usually employed as a differential sensor, that is, it detects wavefront variations with respect to a reference recorded at the beginning of operation. It can be shown [8] that the phase RoC seen by a differential sensor is proportional to the RoC on its conjugate plane through the inverse square of optical magnification: $R o C_{H W S}=\frac{R o C_{c o n j}}{M_{o p t}^{2}}$. In the differential mode of operation, HWS shows an impressive accuracy. An RMS floor noise as low as $0.1 \mathrm{~nm}$ was demonstrated using 100 averages [7].

The main limit to the accuracy of HWS is due to thermal defocus in the afocal optics and thermal deformation of the Hartmann plate, induced by environmental temperature variations, can significantly affect the measurement. In this case, adding a secondary probe beam at a different wavelength, propagating through the afocal system but reflected back before entering the measured path, would allow the subtraction of thermal defocus effects. This solution will be experimentally investigated as a possible near future upgrade.

\subsection{Actuation: ring heaters}

Ring heaters (RHs) are used to correct for thermoelastic deformation of mirrors in the long resonant cavities $[9,10]$. A RH is also installed around the recycling mirror to allow the control of 
RoC matching in the recycling cavity. The heaters are made of two emitting pyrex rings surrounded by a polished copper shield. The rings are heated by Joule effect, using a helicoidal wrapping of $\mathrm{NiCr}$ flat wire. All the RH materials are suitable for UHV operation.

The coil currents of the two heating rings are counter-propagating. In this way the coupling of the stray magnetic field along the test mass axis with actuation magnets is made negligible.

At full power the Advanced Virgo RH is able to decrease the mirror RoC by $100 \mathrm{~m}$ over a cold value of $1.5 \mathrm{~km}$. According to thermo-mechanical simulations, the achieved actuation gain is $\frac{d R o C}{d P}=-0.93 \mathrm{~m} / \mathrm{W}$ with a high degree of sphericity. The transient behaviour of $\Delta \mathrm{RoC}$ shows a typical overshoot, approaching the regime value by less than $10 \%$ in about $10 \mathrm{~h}$. A spherical thermal lens inside the mirror is also created, with a gain $\frac{d\left(f^{-1}\right)}{d P}=810^{-6} \mathrm{~m}^{-1} \mathrm{~W}^{-1}$.

$\mathrm{RH}$ thermal lens proved to be a precise reference to find the location of test mass centre in HWS maps during commissioning. Also, it has been pointed out [11] that acting on the end masses RoC with RH would help in getting rid of parametric instabilities in long cavities. A RH is also foreseen for adaptive tuning of the squeezing filter cavity [12]. RHs are a natural choice also for the tuning of mirror RoC in future detectors, at least for room temperature operation such as in the high frequency xylophone design of the Einstein Telescope [13].

\subsection{Actuation: $\mathrm{CO}_{2}$ laser projectors}

To recover for the aberrated optical path length (OPL) in the input mass a wavefront correction is applied through a proper transmissive fused silica optic called compensation plate $(\mathrm{CP})$, placed before the input mirror. An optimal temperature distribution in the $\mathrm{CP}$ provides the desired OPL correction through thermo-optic effect. To obtain the desired distribution, the $\mathrm{CP}$ surface is heated by a $\mathrm{CO}_{2}$ laser beam suitably shaped; the wavelength of $\mathrm{CO}_{2} \lambda=10.6 \mu \mathrm{m}$ is completely absorbed by silica, thus offering a very efficient heating.

The $\mathrm{CO}_{2}$ source has a maximum power of $50 \mathrm{~W}$ and is installed inside an acoustic enclosure to avoid noise coupling with the environment. The source RIN is reduced between $10 \mathrm{~Hz}$ and 200 $\mathrm{Hz}$ by an in-loop intensity servo system, to make it compliant with the Advanced Virgo sensitivity requirements; besides, a low frequency power stabilization is looped thorugh a piezo acting on the laser cavity.

Cold defects in $\mathrm{RoC}$ of the optics can be recovered using the central heating $(\mathrm{CH})$ projector, that is, a Gaussian spot intensity at the CP centre. The use of a Gassian heating with the same spotsize as the main interferometer beam is also good practice to maintain the thermal status of the detector when a cavity unlock happens, allowing a fast recovery of the locking.

The thermal lens due to coating absorption in the input masses can be compensated using an optimized axi-symmetric heat distribution on CPs that is obtained as a sum of two ring-shaped intensity patterns [6]. Each ring is produced using an axicon lens, then the two rings are superimposed on an object plane. Hence, the object is imaged onto the CP by a suitable lens. This arrangement is called Double Axicon System (DAS) and allows the power in the two rings to be separately controlled. Also, a lens is placed before the axicon to get the desired ring thickness. Both the lens and the axicon are mounted on remotely controlled translation stages, so that the ring shape can be tuned online.

To tackle the residual, asymmetric distortion, the TCS baseline solution is the so called Scanning System (SS) [14]. SS consists in a Gaussian spot that is scanned along a raster pattern on 
the $\mathrm{CP}$. Fast steering (up to several $\mathrm{kHz}$ step frequency) is provided by galvanometric mirrors. The power delivered on each spot is controlled through an AOM. The desired asymmetric intensity pattern is then obtained as the sum of all the spots: the slow thermal diffusion in silica performs a temporal low-pass filtering of the pattern repetitons. The optimal heating for a given distortion is obtained thorugh an iterative approach that uses FEM simulations [6]. Then, the intensity for each spot is computed via a pseudo-inverse matrix approach.

Scan frequency and step frequency are within the Advanced Virgo sensitivity band, so that SS could give rise to coupled noise through radiation pressure or temperature fluctuations. Other approaches are under investigation to increase the scan rapidity, such as acousto-optic deflectors. However, a DC beam shaping would completely remove the issue: therefore, the reconstruction of the heating pattern using deformable mirrors (DM) is being studied in Tor Vergata lab. A $12 \times 12$ piezo actuators DM from Adaptica [15] with $1.5 \mu \mathrm{m}$ stroke is currently under characterization to be employed in a closed loop configuration based on Gerchberg-Saxton algorytm [16].

However, precise beam shaping largely depends on the goodness of the laser output. Non gaussianities in the TCS $\mathrm{CO}_{2}$ beam have been partially removed by spatially filtering the beam through a pinhole. Nonetheless, to improve the laser beam, a mode cleaner for a $15 \mathrm{~W} \mathrm{CO}_{2}$ source has been designed in Tor Vergata. Since no Faraday isolator is available for that wavelength, a compact ( $1 \mathrm{~m}$ round trip) triangular cavity design is adopted. Also single mode fibres for high power have been considered.

\section{A test facility for the TCS strategies}

A facility for testing methods and approaches of the TCS has been developed in Tor Vergata, and it is called TeTis (Testing TCS integrated strategies). It encompasses a scaled down version of Advanced Virgo test mass, with compensation plate and RH correspondingly resized, all placed in a vacuum chamber with optical and $\mathrm{ZnSe}$ windows for incoming SLED and $\mathrm{CO}_{2}$ beams. The design of TeTis is largely flexible, and in the basic form includes wavefront measurement in transmission and in reflection. Moreover, a dedicated $\mathrm{CO}_{2} 9 \mathrm{~W}$ source is used to produce $\mathrm{CH}$ and DAS projectors. TeTis has been extensively used to test the reliability of FEM simulations with respect to $\mathrm{RH}$ or $\mathrm{CH}$ induced thermal lens or RoC variation. It is the ideal environment to study alternative or innovative solutions, and to record parameters that are not accessible in Advanced Virgo, such as temperatures and thermal defocus.

Studies on HWS thermal effects profited from the TeTis environment. A system for the temperature control of the HWS plate has been designed and tested in the facility, using Peltier cells as actuators. The control performance was measured in $0.01^{\circ} \mathrm{C}$ RMS residual temperature variation.

Also in TeTis it has been possible to solve a problem related to DAS quality. In the TCS, the rings after axicon are superimposed using a thin film polarizer (TFP) at $45^{\circ}$. Of the two rings, the one that is transmitted through the TFP shows a relevant deformation, mainly as a dipolar power imbalance. The effect is related to the large divergence of the ring-shaped beam entering the TFP. To solve this, the beam is collimated and then the divergence is recovered after the TFP, by placing a pair of lenses straddling the TFP. This system has been tested in TeTis and proved to be able to recover completely the shape of the transmitted ring. 
TeTis is being currently employed to study the thermal lens compensation using an optimized DAS projector.

\section{Conclusions}

The Thermal Compensation System for Advanced Virgo has been presented, highlighting the methods employed for the sensing and correction of wavefront distortion in the detector. While the system is basically conceived to work in a closed loop, the versatility of the elements composing the system make it possible a wide range of applications in diagnostics, actuation, adaptive tuning of the optical layout and commissioning needs. The viability of upgrades and enhancements of TCS in view of future detectors has also been briefly discussed. In any case, the flexibility of concepts, within the requirements dictated by applications, will be a key feature of adaptive optics in future detectors. The availability of a test facility for new approaches and techniques, such as TeTis in Tor Vergata lab, is a fundamental asset for the study of present and next generation solutions.

\section{References}

[1] B. P. Abbott et al., Observation of Gravitational Waves from a Binary Black Hole Merger, PRL 116, 6 (2016).

[2] J. Aasi et al., Advanced LIGO, CQG 32, 7 (2015).

[3] Acernese F. et al., Advanced Virgo: a second-generation interferometric gravitational wave detector, $C Q G$ 32, (2015).

[4] B. P. Abbott et al., Multi-messenger Observations of a Binary Neutron Star Merger, ApJ 848, 2 (2017).

[5] A. Rocchi et al., Thermal effects and their compensation in Advanced Virgo, JPCS 363, (2012).

[6] The Virgo collaboration, Advanced Virgo technical design report, VIR-0128A-12, (2012).

[7] A. Brooks, Hartmann wavefront sensors for advanced gravitational wave interferometers, $\mathrm{PhD}$ Thesis, University of Adelaide, (2007).

[8] I. Nardecchia, Control of optical aberrations in advanced interferometric gravitational wave detectors, PhD Thesis, Università degli Studi di Roma La Sapienza and Università degli Studi di Roma Tor Vergata, (2016).

[9] H. Lück et al., Thermal correction of the radii of curvature of mirrors for GEO 600, CQG 21, (2004).

[10] J. Ramette et al., Analytical model for ring heater thermal compensation in the Advanced Laser Interferometer Gravitational-wave Observatory, Applied Optics, 55, 10 (2016).

[11] M. Evans et al., Thermal tuning of optical cavities for parametric instability control, JOSA 24, (2007).

[12] J. Miller et al. Prospect for doubling the range of Advanced LIGO, PRD 91, 06 (2015).

[13] M. Punturo et al., The Einstein Telescope: a third-generation gravitational wave observatory, CQG 27, 19 (2010).

[14] R. C. Lawrence, Active wavefront correction in laser interferometric gravitational wave detectors, PhD Thesis, MIT, (2003). 
[15] www.adaptica.com .

[16] R. W. Gerchberg and W. O. Saxton, A practical algorithm for the determination of the phase from image and diffraction plane pictures, Optik 35, 19 (1972). 